\title{
Towards GHz switching in SOI light emitting diodes
}

\author{
Vidhu Puliyankot, Giulia Piccolo, Raymond J.E. Hueting, Senior Member, IEEE and \\ Jurriaan Schmitz, Senior Member, IEEE
}

\begin{abstract}
In this work we study the switching behavior of silicon-on-insulator light-emitting diodes. Through the comparison of various device geometries we establish the dimensional dependence of the switching speed of the light-emitting diode. TCAD simulations are in line with the experimental results. Our findings indicate that on-off keying up to $\mathrm{GHz}$ frequencies should be feasible with such diodes, although a design optimized for higher frequency operation will exhibit a reduced light emission efficiency.
\end{abstract}

Index Terms-Light emitting diodes, modeling, quantum efficiency, silicon photonics, simulation.

\section{INTRODUCTION}

CMOS offers a technology base with a spectacular combination of technological sophistication and efficient economics [1]. Adding photonics to standard CMOS offers new functionalities such as: high data rate on-chip interconnections [2], [3], photonic links between processor and memory [4], [5], and optical sensor applications [6], [7]. Arguably, the addition of photonic functionality to digital logic is of particular interest for high-performance systems.

Electronic-photonic integration needs a CMOS-compatible, efficient and fast light source; waveguide; and photodetector. So far scientists have been able to develop high quality waveguides, detectors and optocouplers in CMOS compatible technologies [8]-[10]. An efficient, integrated light source remains to be found to complete the toolbox. Avalanchebased light emission can be achieved in a standard silicon technology, reaching high modulation speeds beyond $20 \mathrm{GHz}$ be it with a quantum efficiency in the $10^{-7}-10^{-5}$ range [11][13]. In this article we investigate the switching speed of the more efficient forward-biased Si Light-Emitting Diode (LED). Forward biased Si LEDs emitting around $1150 \mathrm{~nm}$ wavelength have shown external efficiencies close to $1 \%$ in bulk silicon, much higher than long-anticipated earlier [14]. Light emission in FinFET technologies has also been reported recently [15], [16]. Standard silicon platforms therefore inherently offer light emission capability. The switching speed of such LEDs has however hardly been studied in contrast to direct-bandgap LED switching [17], with the notable exception of [18].

In this work we measure optical switching in lateral silicon on insulator (SOI) p-i-n LEDs. Conventional diode designs are compared to narrow-injector layouts [19], meant to decouple the carrier injection level from the diode current. TCAD simulations are used to identify the limitations in the switching speed in these devices.

The authors are with the MESA+ Institute for Nanotechnology, University of Twente, P.O. Box 217, 7500AE, Enschede, The Netherlands (email: j.schmitz@utwente.nl).

This work was financially supported by Dutch Ministry of Economic Affairs in the framework of the MEMPHIS project.
This paper is outlined as follows. In section II we describe the background theory needed for this work. In sections III and IV we discuss the experimental material and analyze the obtained results. In section $\mathrm{V}$ we further analyze the results with TCAD simulations. Finally, we come up with the conclusions in section VI.

\section{THEORY}

When a forward bias is applied to a p-i-n diode, charge carriers are injected into the active (intrinsic) region. These carriers may recombine, possibly through a (phonon-assisted) radiative process. The charge carrier concentration inside the active region determines the radiative recombination rate $R_{\text {Rad }}$ according to [20]-[23]:

$$
R_{\mathrm{Rad}}=B_{\mathrm{Rad}} \cdot\left(p n-n_{\mathrm{i}}^{2}\right),
$$

where $B_{\mathrm{Rad}}$ is the radiative recombination coefficient, $p$ and $n$ are the hole and electron concentration respectively; and $n_{\mathrm{i}}$ is the intrinsic carrier concentration. A higher forward bias $V_{\mathrm{D}}$, hence injection, gives a higher $R_{\text {Rad }}$ through the higher $p n$ product and therefore more light output, following

$$
p n=n_{\mathrm{i}}^{2} \cdot \exp \left(\frac{E_{\mathrm{FN}}-E_{\mathrm{FP}}}{k T}\right)=n_{\mathrm{i}}^{2} \cdot \exp \left(\frac{V_{\mathrm{D}}}{u_{T}}\right) .
$$

Here, $u_{T}$ is the thermal voltage, $E_{\mathrm{FN}}, E_{\mathrm{FP}}$ are the quasiFermi levels in the active region, $k$ is Boltzmann's constant and $T$ the absolute temperature.

Upon a changing diode bias, the temporal evolution of the term $\left(p n-n_{i}^{2}\right)$ directly determines the light emission over time. The device's optical switching speed is therefore governed by the rate of change of the $p n$ product. The total stored charge inside the active region must change upon switching, by drift, diffusion and/or generation/recombination.

\section{A. On-switching}

When a p-i-n diode turns on due to a step in the applied voltage from negative to positive bias, the concentration of electrons and holes in the intrinsic region will increase exponentially with voltage. Charge is supplied from the $\mathrm{p}$ and $\mathrm{n}$ regions and fills the intrinsic region by diffusion. The time needed for such a process, i.e. the rise time, can be approximated as

$$
\tau_{\mathrm{r}}=\left|\frac{\Delta Q}{\Delta I}\right| \approx \frac{q A n_{o n} L}{\overline{I_{\mathrm{r}}}},
$$

with $\Delta Q$ is the change in the stored charge in the active region, $\Delta I$ is the (transient) displacement current of this charge, $n_{\text {on }}$ is the electron concentration in the on-state, $L$ is the active region length, $A$ is the device area, and $\overline{I_{\mathrm{r}}}$ is the average 
rise (or forward) current flowing through the diode during the transient.

$\bar{I}_{\mathrm{r}}$ comprises a mix of diffusion (low bias) and drift (high bias) currents. No analytical solution is available for the current development over time, and therefore numerical calculations are required, as done in this work using TCAD.

Equation 3 indicates that $\bar{I}_{\mathrm{r}}$ should be as high as possible for fast switching. However, this must be enforced with a larger forward voltage. Generally this leads to a faster rise of $\Delta Q$ than $\bar{I}_{\mathrm{r}}$. As a result, a higher speed is obtained at lower forward voltage, as will be shown later.

\section{B. Off-switching}

At off-switching, excess charge in the intrinsic region should be removed. For the fall time $\tau_{\text {fall }}$ the same relation holds as for $\tau_{\mathrm{r}}$ described above. The main difference with the onswitching process is that the average fall (or reverse) current $\bar{I}_{\mathrm{f}}$ is basically a drift current. Consequently, $\tau_{\text {fall }} \ll \tau_{\mathrm{r}}$ and therefore $\tau_{\text {fall }}$ can be ignored in most cases as done in this work.

Assuming that the switching response of an LED is characterized by a single time constant $\tau$ and the LED is biased for on-off keying, its time-averaged brightness will reach $3 \mathrm{~dB}$ attenuation at a frequency given by $f_{3 \mathrm{~dB}} \approx \sqrt{3} /(2 \pi \tau)$, assuming a single pole model [17]. We make use of this principle to quantify the switching speed of a diode by time-integrated optical intensity measurements, as detailed in Section IV. The Appendix offers a more elaborate treatment of on- and offswitching in $\mathrm{p}-\mathrm{i}-\mathrm{n}$ diodes.

\section{Efficiency vs. speed}

The internal efficiency $\eta_{\mathrm{IQE}}$ is defined by the ratio of the total amount of photons created per second and the total amount of injected charge carriers per second [17]. Hence, it can be stated that [24]

$$
\eta_{\mathrm{IQE}}=\frac{I_{\mathrm{RAD}}}{I_{\mathrm{tot}}}
$$

with $I_{\mathrm{RAD}}$ the radiative recombination current, which is basically a measure for the photon production, and $I_{\text {tot }}$ the total current. $I_{\mathrm{RAD}}$ is essentially determined by silicon material properties such as $B_{\text {Rad }}$ (see also Eq. (1)) and is therefore governed by the injection level. For obtaining a high $\eta_{\mathrm{IQE}}$ one therefore aims to reduce $I_{\text {tot }}$ by device design at a given $p n$ product. This can be done by employing wide bandgap materials in the injector regions (e.g., [17], [24], [25]) or by adopting narrow injector regions [19], see also Fig. 1(c).

For a given $p n$ product, $I_{\text {tot }}$ should be small to achieve high $\eta_{\mathrm{IQE}}$. A small $I_{\text {tot }}$ leads to a smaller $I_{r}$ and therefore leads to a longer $\tau_{\text {rise }}$ at fixed $Q$. This implies that there is a trade-off between the internal efficiency and switching speed.

\section{DEVICE FABRICATION}

Fig. 1 shows a schematic cross-section of the SOI LEDs under consideration. We studied two types of LEDs, a conventional p-i-n diode (Fig.1(b)) and a narrow-injector p-i-n diode (Fig.1(c)). For the narrow injector diode we kept the active

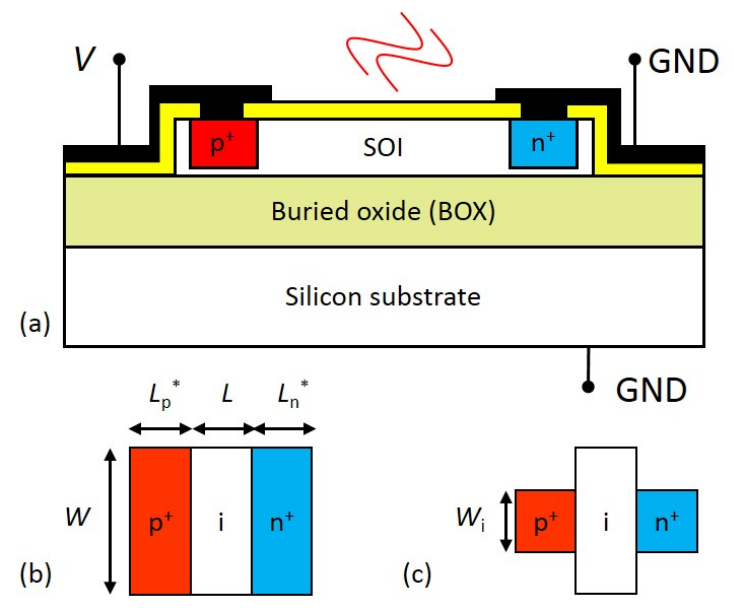

Fig. 1. Schematic layout of the SOI light emitting diode with (a) the crosssection and (b) the top view of the conventional diode. Optical switching was studied for 5 and $10 \mu \mathrm{m}$ active region lengths $(L)$ and with $L_{p}^{*}=$ $L_{n}^{*}=10 \mu \mathrm{m}$. $W_{\mathrm{i}}=W=100 \mu \mathrm{m}$. (c) Shows the top view of the narrow injector LED used for studying the injection dependency. $W_{\mathrm{i}}=10 \mu \mathrm{m}$ and $L=5 \mu \mathrm{m}$.

region dimensions constant and reduced the injector width of the LED from $100 \mu \mathrm{m}$ to $10 \mu \mathrm{m}$. The basic idea of the narrow injector LED [19] is to vary the injection condition as discussed further in this paper.

The active region length $L$ was varied between 5 and $10 \mu \mathrm{m}$. The devices were fabricated in high quality SOI substrates ( $\mathrm{p}$ type, $\rho=14-22 \Omega \cdot \mathrm{cm}), t_{\mathrm{Si}}=300 \mathrm{~nm}$, on top of a $400 \mathrm{~nm}$ buried oxide layer (BOX). The SOI islands were formed by wet etching and subsequently insulated by a $10-n m$ thermal oxide layer. The injector regions (i.e. the $\mathrm{p}^{+}$and $\mathrm{n}^{+}$regions) were realized by implantation of $6 \cdot 10^{15} \mathrm{~cm}^{-2}$ of $\mathrm{B}^{+}$and $5 \cdot 10^{15} \mathrm{~cm}^{-2}$ of $\mathrm{P}^{+}$at 30 and $50 \mathrm{kV}$ respectively. Dopants were activated with a $5 \mathrm{~s}$ rapid thermal anneal at $900{ }^{\circ} \mathrm{C}$, after deposition of $30 \mathrm{~nm}$ PECVD silicon oxide (at $150{ }^{\circ} \mathrm{C}$ ). Contact windows were defined and $\mathrm{Al} / \mathrm{Si}(1 \%)$ contacts to the $\mathrm{p}^{+}$and $\mathrm{n}^{+}$regions were sputtered. The devices were sintered at $400{ }^{\circ} \mathrm{C}$ in forming gas for 10 minutes.

Based on earlier studies [26], [27], diodes fabricated in this manner exhibit typical internal quantum efficiencies around $10^{-4}$ and a linear current-intensity relationship. The light emission peaks around $1150 \mathrm{~nm}$, as follows from the $1.12 \mathrm{eV}$ silicon band gap.

\section{Measurements}

Fig. 2 shows the DC $I-V$ measurements of the conventional and reduced injector LEDs with $L=5 \mu \mathrm{m}$ and $10 \mu \mathrm{m}$. The $I$ $V$ measurements have been carried out using a Keithley 4200 semiconductor characterization system and a Karl Süss PM8 probe station. The $I-V$ curve of the diodes indicate that the series resistance increases for an increasing $L$. The $5 \mu \mathrm{m}$ long diode has a series resistance of $39 \Omega$ and the $10 \mu \mathrm{m}$ one has a resistance of $89 \Omega$. Generally the series resistance was extracted from the Gummel plot (Fig. 2) by taking the voltage drop between the extrapolated exponential curve and the actual $I-V$ curve at the same current level. As we reduce the injector width $W_{\mathrm{i}}$ from 100 to $10 \mu \mathrm{m}$ for $L=5 \mu \mathrm{m}$ the total current flowing through the diode diminishes for the same 


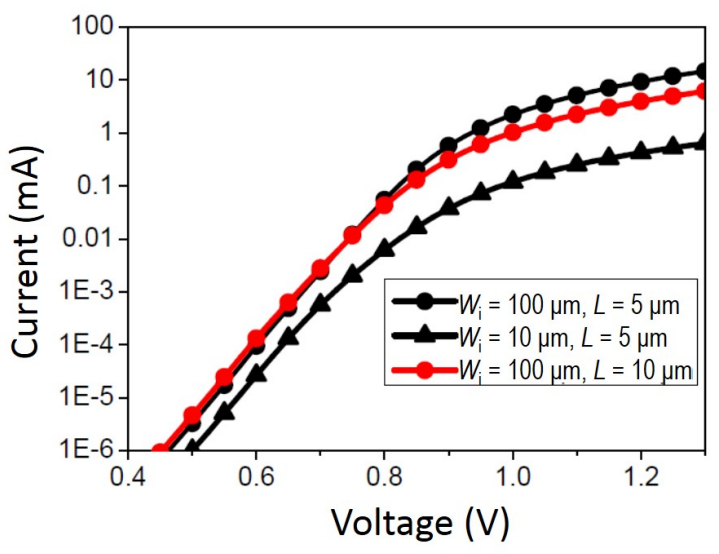

Fig. 2. Measured $I-V$ curve of diodes. For an increase in active length $L$ from $5 \mu \mathrm{m}$ to $10 \mu \mathrm{m}$ an increased resistance is observed at high injection.

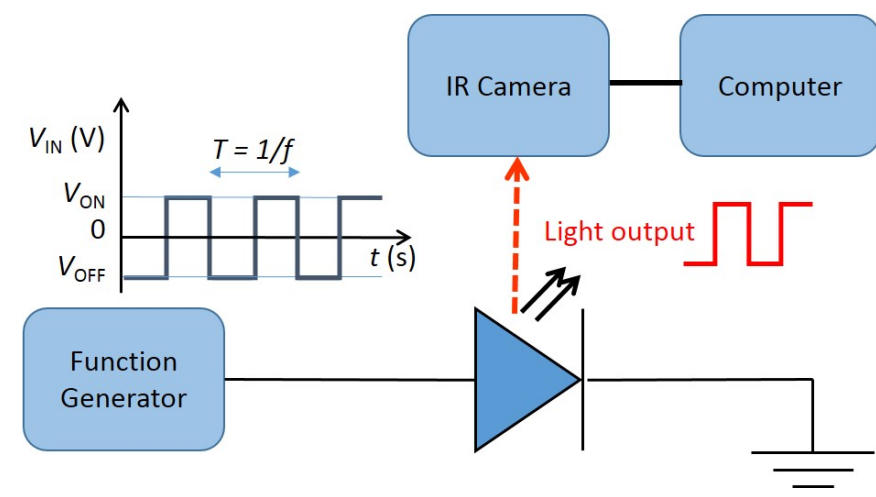

Fig. 3. Schematic layout of the measurement setup. For the measurements, square shaped voltage signals for different amplitudes $V_{\mathrm{ON}}$ and $V_{\mathrm{OFF}}$ at various frequencies were used as an input. The duty cycle was kept constant at 0.5 .

applied voltage, both in low and high injection. The drop at low injection is because the total current is limited by the injector current via diffusion, since it is proportional to $W_{\mathrm{i}}$. As a result, for the same current, the applied voltage, hence the $p n$ product increases (Eq.(2)) and so does the efficiency [19], [24]. At high injection, the narrow-injector LED shows a much higher series resistance of approximately $816 \Omega$.

The emitted light from the diodes biased in forward was characterized with a measurement setup as sketched in Fig. 3. Square wave pulses with varying amplitudes of the input voltage, $V_{\mathrm{ON}}, V_{\mathrm{OFF}}$ were used as indicated in the same figure. The light emitted from the diode was collected by a microscope and passed onto a cooled infrared camera. The camera contains a matrix of $(256 \times 320)$ InGaAs photodiodes which are sensitive in the $0.9 \mu \mathrm{m}-1.7 \mu \mathrm{m}$ wavelength range. The pixel information was processed by custom software (Xcontrol) [28].

Fig. 4 shows infrared images of some diodes illuminated with a weak external light (to visualize the test structures). The metal pads for providing the contacts to the LED are clearly visible. Fig. 4(a) shows the conventional diode in action; (b) shows the diode with narrow injectors. Fig. 5 shows infrared images of the conventional diodes for various square wave pulses. As we increase the frequency of the square wave, the time-integrated
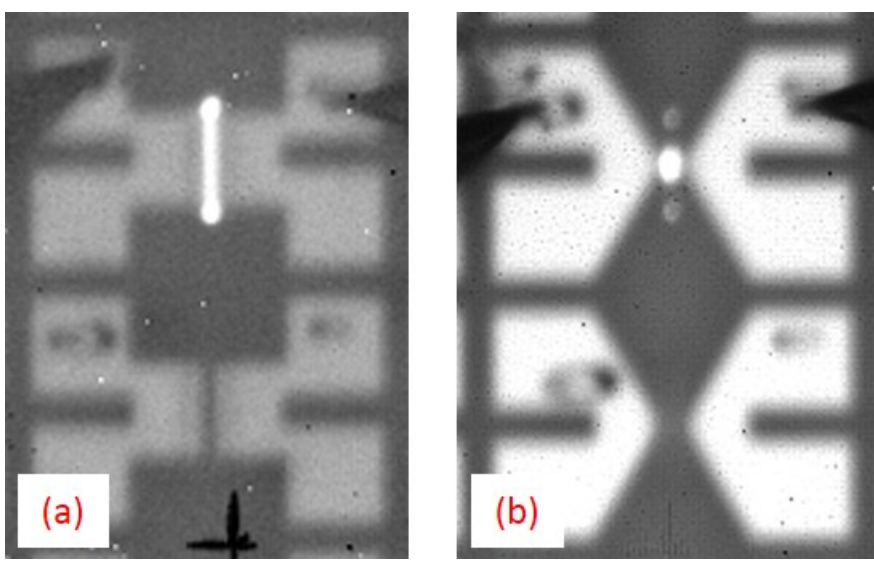

Fig. 4. Top view infrared images of the investigated diodes, exposed to weak external light to visualize the bond pads and probe needles; as obtained from the (a) conventional and (b) narrow injector LED.

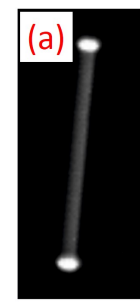

$1 \mathrm{kHz}$

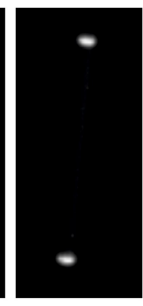

$10 \mathrm{MHz}$

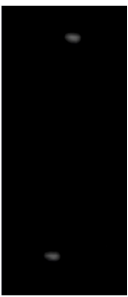

$40 \mathrm{MHz}$

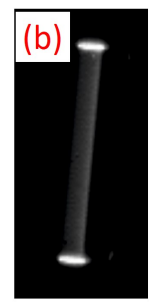

$1 \mathrm{kHz}$

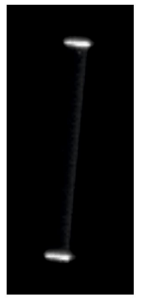

$5 \mathrm{MHz}$

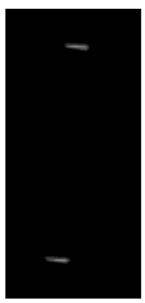

$30 \mathrm{MHz}$
Fig. 5. Top view infrared images of conventional-injector diodes with (a) $L=5 \mu \mathrm{m}$ and (b) $L=10 \mu \mathrm{m}$ at various test frequencies. Both devices have $W_{\mathrm{i}}=100 \mu \mathrm{m}$; the integration time is the same for all images.

light emission from these diodes gradually decreases in the few-MHz regime.

The integrated electroluminescence (EL) for these devices is shown in Fig. 6 as a function of frequency and bias. For higher $V_{\text {ON }}$ higher electroluminescence is observed. The results show that the light output reduces as the switching frequency is increased above $100 \mathrm{kHz}$. For $L=5 \mu \mathrm{m}$, at a frequency of 8 $10 \mathrm{MHz}$ the emitted light output reaches $f_{3 \mathrm{~dB}}$. For $L=10 \mu \mathrm{m}$ the $f_{3 \mathrm{~dB}}$ is around 5-6 MHz. As $L$ is increased, at high injection the series resistance increases by a factor of two (see Fig. 2). This in turn reduces the switching speed (See Eq. (10)).

To investigate whether the injection condition determines the switching speed of the LED, we performed switching measurements in the narrow injector LEDs [19]. Fig. 7 shows top view infrared images of the narrow injector LED at different frequencies. Also here, as the frequency increases, the light output reduces. A reduced switching speed of $2 \mathrm{MHz}$ is observed (see Fig. 8). As stated before, by reducing the injector width, the current flow through LED reduces. Since the stored charge $Q$ (or $p n$ product) is kept constant for the same $V_{\mathrm{ON}}$ (same active region), as the current drops for narrow width, the switching delay times increase for a narrow-injector width (see Appendix). Consequently, the speed of operation reduces. This indicates that the injection condition is an important factor determining the speed of operation.

The narrow injector LEDs are more efficient than the conven- 

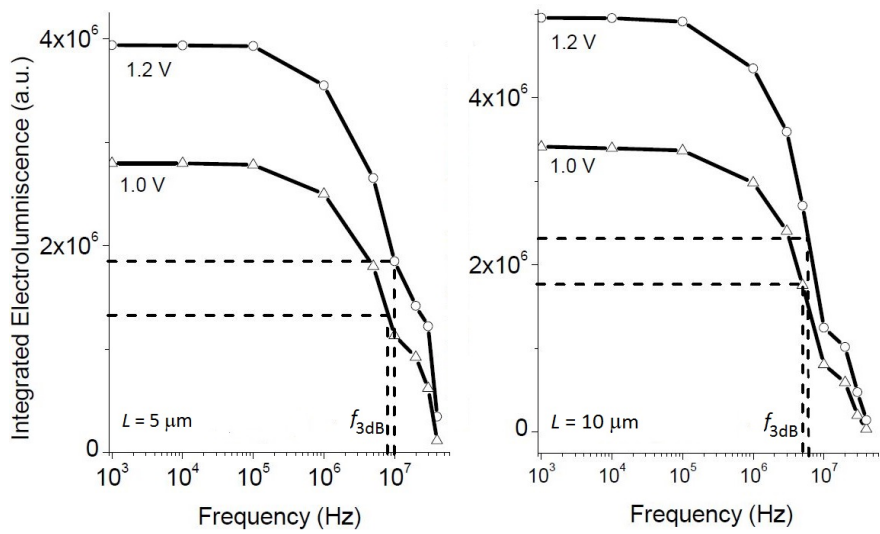

Fig. 6. Measured integrated electroluminescence of the diodes (integration time $t=1 \mathrm{~s}$ ): (left) for $L=5 \mu \mathrm{m}$, the obtained $f_{3 \mathrm{~dB}}$ values are $8 \mathrm{MHz}$ for $V_{\mathrm{ON}}=1 \mathrm{~V}$ and $10 \mathrm{MHz}$ for $V_{\mathrm{ON}}=1.2 \mathrm{~V}$. (Right) For $L=10 \mu \mathrm{m}$, the obtained $f_{3 \mathrm{~dB}}$ values are $5 \mathrm{MHz}$ for $V_{\mathrm{ON}}=1 \mathrm{~V}$ and $6 \mathrm{MHz}$ for $V_{\mathrm{ON}}=1.2 \mathrm{~V}$. $V_{\mathrm{OFF}}$ was kept at $-1.0 \mathrm{~V}$ for all measurements.

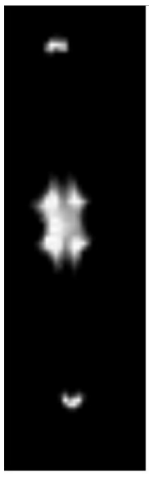

$1 \mathrm{kHz}$

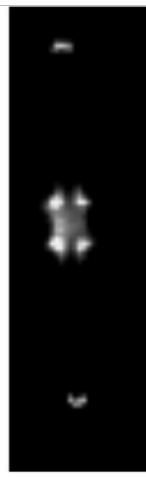

$2 \mathrm{MHz}$

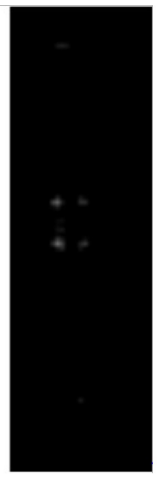

$5 \mathrm{MHz}$
Fig. 7. Top view infrared images of the lateral narrow injector $(L=5 \mu \mathrm{m}$ $\left.W_{\mathrm{i}}=10 \mu \mathrm{m}\right)$ diode at various frequencies using $V_{\mathrm{ON}}=1.2 \mathrm{~V}$ and $V_{\mathrm{OFF}}=-$ $1.0 \mathrm{~V}$.

tional diodes [19], [24]. However, as discussed in Section II.C, the optical switching speed reduces when the efficiency increases. This trade off between efficiency and speed is further evaluated via simulations in section V.C.

\section{TCAD SIMULATION}

To gain insight into the switching behaviour, transient two-dimensional (2D) TCAD [29] simulations have been performed. The simulations apply the Boltzmann approximation with Philips' unified mobility model [30], a doping-induced bandgap narrowing model [31], and default recombination models (unless stated otherwise). The following $\mathrm{Si}$ recombination parameters were used: $B_{\text {Rad }}=10^{-14} \mathrm{~cm}^{3} \mathrm{~s}^{-1}[32], \quad \tau_{\mathrm{SRH}}=2.5 \cdot 10^{-5} \mathrm{~s}$, $C_{n}=1.83 \cdot 10^{-31} \mathrm{~cm}^{6} \mathrm{~s}^{-1}$ and $C_{p}=2.81 \cdot 10^{-31} \mathrm{~cm}^{6} \mathrm{~s}^{-1}$ [33]. All simulations in this work were performed at ambient temperature $(T=300 \mathrm{~K})$. The uniformly $10^{19} \mathrm{~cm}^{-3}$ doped $\mathrm{p}^{+}$and $\mathrm{n}^{+}$injector regions have a length of $10 \mu \mathrm{m}$.

Fig. 9(a) shows typical transient simulation results. The $R_{\mathrm{Rad}}$, which is a measure of light output, is integrated over the $2 \mathrm{D}$ active region and is plotted for different frequencies. Fig. 9(b) shows the $R_{\text {Rad }}$ from Fig. 9(a) integrated over a $1 \mathrm{~s}$ time

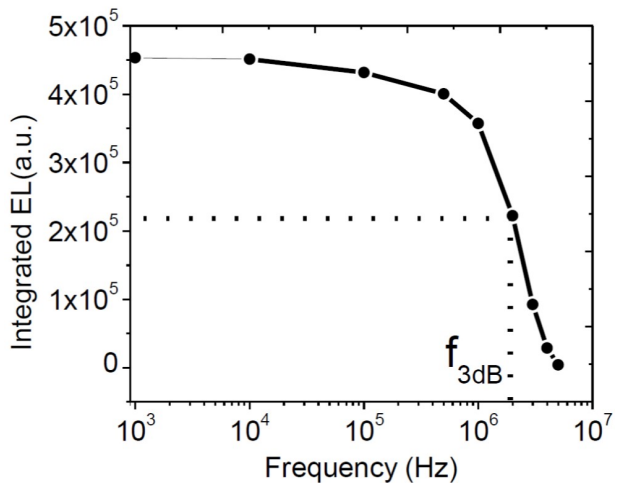

Fig. 8. Measured integrated electroluminescence of the narrow injector $\left(L=5 \mu \mathrm{m}, W_{\mathrm{i}}=10 \mu \mathrm{m}\right)$ diodes. $f_{3 \mathrm{~dB}}=2 \mathrm{MHz}$ for $V_{\mathrm{ON}}=1.2 \mathrm{~V}$ and $V_{\mathrm{OFF}}=-1.0 \mathrm{~V}$.

period. At higher frequencies, mainly the slow turn-on of the diode reduces the integrated $R_{\text {Rad }}$ and hence the light output. This supports and quantifies the assumption of $\tau_{\text {fall }} \ll \tau_{\text {r }}$ discussed in Section II. The $f_{3 \mathrm{~dB}}$ is found to be between 8 and $10 \mathrm{MHz}$, in good agreement with the experiments. For $L=10 \mu \mathrm{m}$, an $f_{3 \mathrm{~dB}}$ of $3 \mathrm{MHz}$ is obtained showing the same trend. Table I summarizes our findings.

In the following, we continue to investigate the effect of several transport parameters on the switching performance.

\begin{tabular}{|c|c|c|c|}
\hline $\mathrm{L}(\mu \mathrm{m})$ & $\mathrm{W}(\mu \mathrm{m})$ & $f_{3 \mathrm{~dB}}(\mathrm{MHz})(\exp )$ & $f_{3 \mathrm{~dB}}(\mathrm{MHz})(\mathrm{TCAD})$ \\
\hline 5 & 100 & 8 & 8 \\
\hline 10 & 100 & 5 & 3 \\
\hline 5 & 10 & 2 & - \\
\hline
\end{tabular}

TABLE I

$f_{3 \mathrm{~dB}}$ DATA OBTAINED FROM MEASUREMENTS AND TCAD SIMULATIONS $\left(V_{\mathrm{ON}}=1.0 \mathrm{~V}, V_{\mathrm{OFF}}=-1.0 \mathrm{~V}\right)$.

\section{A. Effect of mobility on switching}

To study the effect of mobility on switching we increased the mobility by a factor of $\simeq 4$ (resembling Ge) while keeping all other parameters fixed (Table II). The transient simulations showed much faster response compared to those reported in Fig. 9. $f_{3 \mathrm{~dB}}$ was increased to $40 \mathrm{MHz}$. This can be attributed to the reduced $R C$ time.

\section{B. Effect of recombination rates on switching}

We also studied the effect of the radiative recombination coefficient $\left(B_{\text {Rad }}\right)$ on the switching speed. Transient TCAD simulations show no effect (Table II) in the speed of operation. However, an increase in the a light output (Eq. (1)) is observed as $B_{\mathrm{Rad}}$ values are increased. The $f_{3 \mathrm{~dB}}$ remains in the $10 \mathrm{MHz}$ range even when $B_{\text {Rad }}$ values comparable to those of III-V compounds are used. This is because in Si LEDs, the radiative current component at high injection conditions does not affect the current flow through, and stored charge in, the diode [24], hence the $R C$ time. In direct bandgap based LEDs however the $\tau_{\mathrm{r}}$ is governed by radiative recombination [17]. 

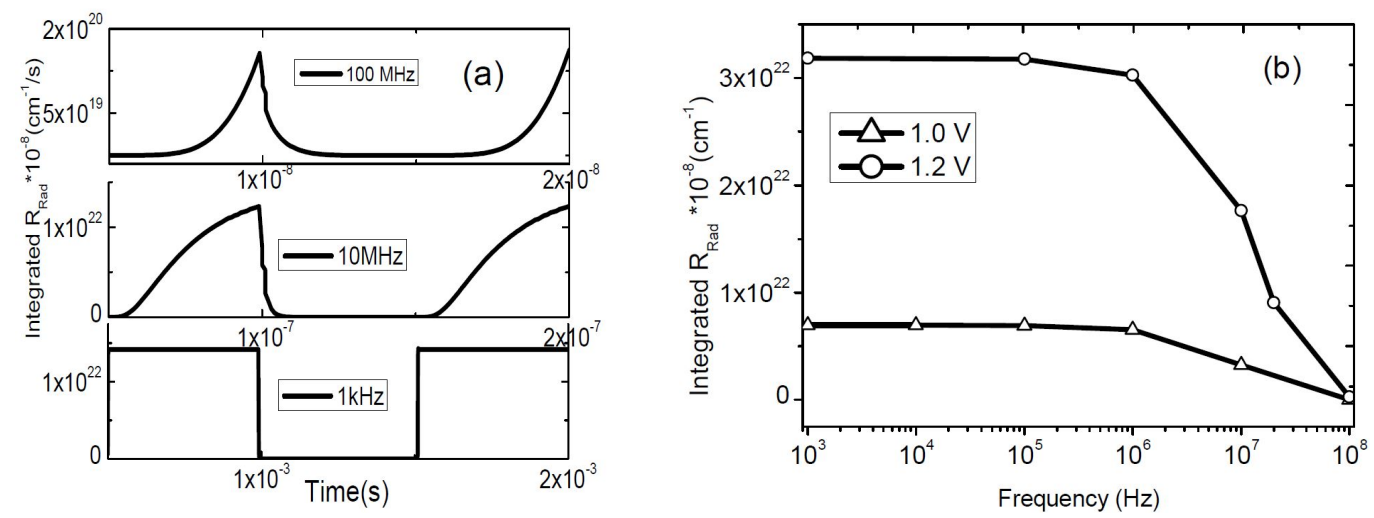

Fig. 9. (a) Transient simulation results showing integrated $R_{\mathrm{Rad}}$ from the active region for $V_{\mathrm{ON}}=1.0 \mathrm{~V}$. The integrated $R_{\mathrm{Rad}}$ is plotted for $1 \mathrm{kHz}, 10 \mathrm{MHz}$ and $100 \mathrm{MHz}$. (b) Simulated light output for a time period of $1 \mathrm{~s}$. The curves are obtained by integrating $R_{\mathrm{Rad}}$ over (2D) space and time. Cutoff frequencies $f_{3 \mathrm{~dB}}$ of 8 and $10 \mathrm{MHz}$ are found for $V_{\mathrm{ON}}=1.0 \mathrm{~V}$ and $1.2 \mathrm{~V}$ respectively. The simulations were performed for $L=5 \mu \mathrm{m}$ with $V_{\mathrm{OFF}}=-1.0 \mathrm{~V}$.

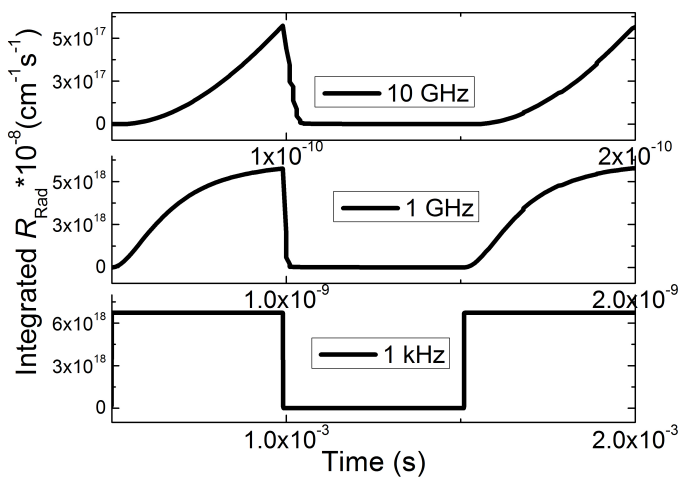

Fig. 10. Transient simulation results at low injection showing integrated $R_{\mathrm{Rad}}$ from the active region. Integrated $R_{\mathrm{Rad}}$ is plotted for $1 \mathrm{kHz}, 1 \mathrm{GHz}$, and $10 \mathrm{GHz}$. A much faster response from the diode is observed compared to results shown in Fig. 9. The simulations were performed for $L=0.5 \mu \mathrm{m}$, $V_{\mathrm{ON}}=0.80 \mathrm{~V}$ and $V_{\mathrm{OFF}}=-1.0 \mathrm{~V}$.

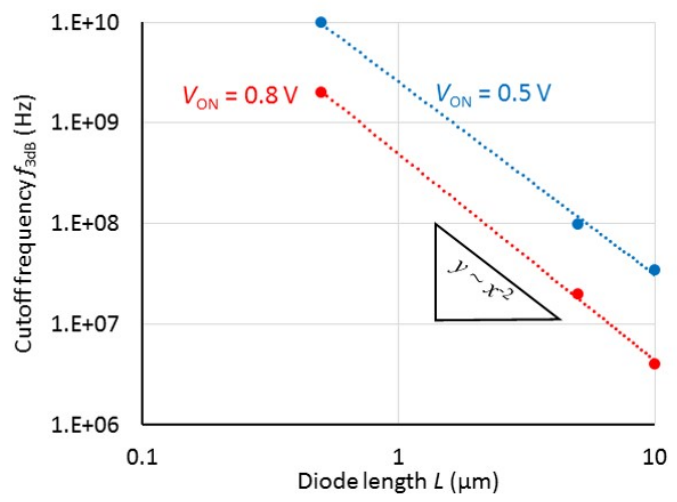

Fig. 11. Simulated cutoff frequency $f_{3 \mathrm{~dB}}$ for various diodes at two injection conditions. For $L=0.5 \mu \mathrm{m}$ the cutoff frequency reaches the GHz-range. For lower applied voltages $\left(V_{\mathrm{ON}}\right)$, the $f_{3 \mathrm{~dB}}$ increases. The dashed lines are power-law fits drawn to guide the eye. The simulations were performed for $V_{\mathrm{OFF}}=-1.0 \mathrm{~V}$.
We also varied the Shockley-Read-Hall lifetime $\tau_{\mathrm{SRH}}$ and the Auger recombination lifetime values and those hardly showed any effect on the switching speed.

\begin{tabular}{|c|c|c|c|}
\hline $\mathrm{L}(\mu \mathrm{m})$ & Mobility $\mu$ & $B_{\mathrm{Rad}}\left(\mathrm{cm}^{3} \mathrm{~s}^{-1}\right)$ & $f_{3 \mathrm{~dB}}(\mathrm{MHz})$ \\
\hline 0.5 & $\mathrm{Si}$ & $10^{-14}$ & 50 \\
\hline 5.0 & $\mathrm{Si}$ & $10^{-14}$ & 8 \\
\hline 5.0 & $\mathrm{Ge}$ & $10^{-14}$ & 40 \\
\hline 5.0 & $\mathrm{Si}$ & $10^{-12}$ & 8 \\
\hline 5.0 & $\mathrm{Si}$ & $10^{-16}$ & 8 \\
\hline
\end{tabular}

TABLE II

$f_{3 \mathrm{~dB}}$ DATA OBTAINED FROM TCAD SIMULATIONS ONLY $\left(V_{\mathrm{ON}}=1.0 \mathrm{~V}\right.$, $\left.V_{\mathrm{OFF}}=-1.0 \mathrm{~V}\right)$.

\section{Optimization}

As the active region length $L$ has a strong effect on the $R C$ delay (see the Appendix), the next step to increase the switching speed would be to reduce $L$. According to our simulations $f_{3 \mathrm{~dB}}$ increases to $50 \mathrm{MHz}$ for a diode with $L=0.5 \mu \mathrm{m}$ at high injection switching $(1.0 \mathrm{~V})$.

Fig. 10 shows the transient simulation for $L=0.5 \mu \mathrm{m}$ at low injection. As the $V_{\mathrm{ON}}$ is reduced to $0.80 \mathrm{~V}$, much faster switching operation is observed and $f_{3 \mathrm{~dB}}$ reaches the $\mathrm{GHz}$ range. Fig. 11 shows $f_{3 \mathrm{~dB}}$ for various active region lengths, indicating an increase in $f_{3 \mathrm{~dB}}$ for reduced device dimensions and injection conditions. These simulations show an $L^{-2}$ dependence. The cut-off frequency reaches the GHz-range for smaller active region lengths.

These simulations clearly indicate that there is trade-off between speed and total light output (or efficiency) [19], [24]. At very low injections, high speed optical switching is possible; however the total light output $\left(R_{\operatorname{Rad}} \propto \exp \left(\frac{V_{\mathrm{D}}}{u_{T}}\right)\right)$ and efficiency will be quite low. As the light output increases, the speed reduces drastically, in line with the theoretical considerations in this article and the narrow-injector LED measurements.

\section{CONCLUSION}

We experimentally showed $10 \mathrm{MHz}$ switching operation in forward biased SOI LEDs, which is much higher than might 
be expected from the radiative lifetime in silicon. TCAD simulations, which are in good agreement with measurements, confirm that the radiative lifetime has a negligible effect on the switching speed. Both measurements and TCAD simulations indicate that reducing the device dimensions can further increase the switching speed. The simulations also show a trade-off between the efficiency and switching speed in the diode. This trade-off is observed in our experiments as highly efficient narrow-injector LEDs showed lower speeds compared to conventional counterparts. Finally, the simulations predict that, for an optimised diode, cutoff frequencies in the $\mathrm{GHz}$ range can be achieved at low injection conditions.

\section{APPENDIX: SWITCHING THEORY}

This section discusses the basic switching theory of a 1D p-i-n diode under the following assumptions:

- The quasi-static approach is used, which means that the wavelength of the electrical signal is much larger than the device dimensions.

- Only the charge build-up/reduction in the active region is considered; the charge in the injector regions is neglected.

- Bulk recombination is ignored, unlike in [34]. For devices with active lengths of $10 \mu \mathrm{m}$ or less this is considered insignificant for the drift-diffusion balance.

Initially, as traditionally done in many text books (e.g., [35]), the small-signal behavior is discussed before diving into the transient (or large-signal) behavior.

\section{A. On-switching}

For switching on the LED the excess charge will be mainly built up via diffusion of charge carriers [24]. The total diffusion current can be written as

$$
I_{\text {dif }}=-q A n_{i}^{2} \cdot\left(\frac{D_{n}}{N_{\mathrm{A}} \cdot L_{\mathrm{p}}}+\frac{D_{p}}{N_{\mathrm{D}} \cdot L_{\mathrm{n}}}\right) \cdot \exp \left(\frac{V_{\mathrm{D}}}{u_{T}}\right),
$$

where $D_{n, p}$ is the diffusion constant, $N_{\mathrm{A}, \mathrm{D}}$ is the doping concentration in the injector regions and $L_{\mathrm{p}, \mathrm{n}}$ is the neutral injector length. Since $V_{\mathrm{D}}$ drops over the active region and there is zero space charge, the excess charge density in the active region can be written as

$$
p=n=n_{i} \cdot \exp \left(\frac{V_{\mathrm{D}}}{2 \cdot u_{T}}\right),
$$

and is practically constant throughout the active region. The excess charge then follows:

$$
Q=-q A n L=-q A n_{i} \cdot \exp \left(\frac{V_{\mathrm{D}}}{2 \cdot u_{T}}\right) L .
$$

At high injection $\left(V_{\mathrm{D}}>0.8 \mathrm{~V}\right.$ as can be established by TCAD and from Fig. 2) the current eventually becomes a drift current described as

$$
I_{\mathrm{d}}=-q A\left(\mu_{p}+\mu_{n}\right) n \frac{V_{\mathrm{D}}}{L},
$$

with $\mu_{p, n}$ the charge carrier mobility.

Combining Equations 7 and 8:

$$
Q=\frac{I_{\mathrm{d}} \cdot L^{2}}{\left(\mu_{p}+\mu_{n}\right) V_{\mathrm{D}}},
$$

as was reported for long, 1D p-i-n diodes [34].

For small-signal switching the transit or delay time is defined as:

$$
\tau=\left|\frac{\partial Q}{\partial I}\right|=\left|\frac{\partial Q}{\partial V_{\mathrm{D}}}\right| \cdot\left|\frac{\partial V_{\mathrm{D}}}{\partial I}\right|=C \cdot R,
$$

with $R$ the differential resistance.

For low injection it can be derived from Eq. (10) that

$$
\tau=\frac{Q}{2 I_{\mathrm{dif}}}=\frac{q A n L}{2 I_{\mathrm{dif}}} \propto L \cdot \exp \left(-\frac{V_{\mathrm{D}}}{2 \cdot u_{T}}\right),
$$

while for high injection

$$
\tau=\frac{Q}{I_{\mathrm{d}}}=\frac{L^{2}}{2 u_{T} \mu} \cdot \frac{1}{\left(1+\frac{V_{\mathrm{D}}}{2 u_{T}}\right)} \approx \frac{L^{2}}{\mu V_{\mathrm{D}}},
$$

with $\mu=\mu_{n}+\mu_{p}$. For both cases the capacitance $C=$ $\partial Q / \partial V_{\mathrm{D}}=-\frac{q A n L}{2 u_{T}}$ with $n$ obtained from Eq. (6). Consequently, at high injection

$$
R=\frac{2 L}{q \mu n A} .
$$

In summary, it can be concluded that $\tau$ is inversely proportional to $I$ and proportional to $L$ or to $L^{2}$, depending on the injection level. Further, since $I_{\mathrm{d}}$ is much higher than $I_{\mathrm{dif}}$, at high injection $\tau$ is much smaller than at low injection.

For analyzing the transient (large-signal) behavior of the p-i-n diode one approach is to adopt the relation for the (smallsignal) capacitance $C$ and combine that with a complete dc model for the diode current in which both drift and diffusion components have been incorporated. In a way this was done in our TCAD simulations. However, for the understanding a qualitative discussion would suffice.

For on-switching the rise time can be expressed as:

$$
\tau_{\mathrm{r}} \approx\left|\frac{Q}{\overline{I_{\mathrm{r}}}}\right|_{V=V_{\mathrm{D}}}=\frac{q A n L}{\overline{I_{\mathrm{r}}}},
$$

with $\overline{I_{\mathrm{r}}}$ is the average rise (or forward) transient current:

$$
\overline{I_{\mathrm{r}}}=\frac{\int_{0}^{T} I_{\mathrm{r}} d t}{T}
$$

where $I_{\mathrm{r}}$ is the actual rise current, $t$ is the time and $T$ is the time period of the transient.

All transit times described in the above equations depend on the same $Q$. Therefore it can be concluded that the actual current $I_{\mathrm{r}}$ at the bias operation-point $V_{\mathrm{D}}$ (small-signal) or the average current $\overline{I_{\mathrm{r}}}$ reaching the final bias setting $V_{\mathrm{D}}$ (transient) cause the differences in the switching frequency. When the device is operated solely in low injection mode $\overline{I_{\mathrm{r}}}$ is a diffusion component that is lower than $I_{\mathrm{dif}}$ at the same $V_{\mathrm{D}}$. When the device is also operated in high injection mode $\overline{I_{\mathrm{r}}}$ comprises both a diffusion and drift component, which is much less than the actual drift current for the same $V_{\mathrm{D}}$. Therefore, typically for a high small-signal switching speed the actual current hence bias should be relatively high, while for a high transient switching speed $\overline{I_{\mathrm{r}}}$ hence $V_{\mathrm{D}}$ should be low. 


\section{B. Off-switching}

For switching off the $\mathrm{p}-\mathrm{i}-\mathrm{n}$ diode, the excess charge should be reduced via drift of charge carriers (or by "charge carrier sweep-out" [17]). The high charge carrier concentration, as described in Eq. (6), and an aiding reverse bias field yield a high drift current for the fall (or reverse) current $\overline{I_{\mathrm{f}}}$. In case of small-signal switching the transit time when switching off the device can then be simply described by Eq. (12). For transient switching a relatively low $\tau_{\text {fall }}$ (fast switching off) is obtained according to

$$
\begin{gathered}
\tau_{\text {fall }} \approx\left|\frac{Q}{\overline{I_{\mathrm{f}}}}\right|_{V=V_{\mathrm{D}}}=\frac{q n L}{\overline{I_{\mathrm{f}}}}, \\
\overline{I_{\mathrm{f}}}=\frac{\int_{0}^{T} I_{\mathrm{f}} d t}{T},
\end{gathered}
$$

where $I_{\mathrm{f}}$ is the actual fall current. $\overline{I_{\mathrm{f}}}$ is simply an average drift current (Eq. (8)) and will be much higher than $\overline{I_{\mathrm{r}}}$ during onswitching while the same amount of built-up charge $Q$ should be reduced to zero. Therefore, it can be concluded $\tau_{\text {fall }} \ll \tau_{\text {r }}$. In addition, for low $V_{\mathrm{D}} I_{\mathrm{f}}$ will drop more than $Q$ since the drift current $\sim Q \cdot V_{\mathrm{D}}$, consequently $\tau_{\text {fall }}$ increases for lower $V_{\mathrm{D}}$.

\section{REFERENCES}

11] L. Tsybeskov, D. J. Lockwood and M. Ichikawa, Silicon Photonics: CMOS going optical, Proc. IEEE, vol. 97(7) pp. 1161-1165, 2009. DOI: 10.1109/JPROC.2009.2021052

[2] R. A. Soref, Silicon-based optoelectronics, Proc. IEEE, vol. 81(12) pp. 1687-1706, 1993. DOI: 10.1109/5.248958

[3] D. A. B. Miller, Device requirements for optical interconnects to silicon chips, Proc. IEEE, vol. 97(7) pp. 1166-1185, Jul. 2009. DOI: 10.1109/JPROC.2009.2014298

[4] C. Batten, A. Joshi, J. Orcutt, A. Khilo, B. Moss, C. Holzwarth, M. Popović, H. Li, H. Smith, J. Hoyt, F. Kärtner, R. Ram, V. Stojanović, and K. Asanović, Building manycore processor-to-DRAM networks with monolithic silicon photonics, IEEE Symposium on High Performance Interconnects, vol. 8, pp. 21-30, 2008. DOI: 10.1109/HOTI.2008.11

[5] C. Sun, M. T. Wade, Y. Lee, J. S. Orcutt, L. Alloatti, M. S. Georgas, A. S. Waterman, J. M. Shainline, R. R. Avizienis, S. Lin, B. R. Moss, R. Kumar, F. Pavanello, A. H. Atabaki, H. M. Cook, A. J. Ou, J. C. Leu, Y.-H. Chen, K. Asanović, R. J. Ram, M. A. Popović, and V. M. Stojanović, Single-chip microprocessor that communicates directly using light, Nature, vol. 528(7583), pp. 534-537, Dec. 2015. DOI: 10.1038/nature 16454

[6] R. V. Nair and R. Vijaya, Photonic crystal sensors: An overview, Progress in Quantum Electronics, vol. 34(3), pp. 89-134, May 2010. DOI: 10.1016/j.pquantelec.2010.01.001

[7] N. Jokerst, L. Luan, S. Palit, M. Royal, S. Dhar, M. Brooke, and T. Tyler, Progress in chip-scale photonic sensing, IEEE Trans. Biomed. Circuits Syst., vol. 3(4), pp. 202-211, Aug. 2009. DOI: 10.1109/TBCAS.2009.2020693

[8] L. Pavesi and D. Lockwood, Eds., Silicon Photonics II. In: Topics in Applied Physics, Vol. 119, Springer; Berlin, Heidelberg, 2011.

[9] H.-C. Lee and C.-K. Liu, Si-based current-density-enhanced light emission and low-operating-voltage light-emitting/receiving designs, Solid-State Electronics, vol. 49(7), pp. 1172-1178, Jul. 2005. DOI: 10.1016/j.sse.2005.04.008

[10] S. Dutta, V. Agarwal, R. J. E. Hueting, J. Schmitz and A. J. Annema, Monolithic optical link in silicon-on-insulator CMOS technology, Optics Express, vol. 25(5), pp. 5440-5446, Mar. 2017. DOI: 10.1364/OE.25.005440

[11] M. du Plessis, H. Aharoni, and L. Snyman, Silicon LEDs fabricated in standard VLSI technology as components for all silicon monolithic integrated optoelectronic systems, IEEE J. Select. Topics Quantum Electron. 8(6) pp. 1412-1419, 2002. DOI: 10.1109/JSTQE.2002.806697

[12] A. Chatterjee, B. Bhuva, and R. Schrimpf, High-speed light modulation in avalanche breakdown mode for Si diodes, IEEE Electron Device Lett., vol. 25, no. 9, pp. 628630, Sep. 2004. DOI: 10.1109/LED.2004.834247
[13] K. Xu, Electro-optical modulation processes in Si-PMOSFET LEDs operating in the avalanche light emission mode, IEEE Trans. Electron Dev. 61(6) pp. 2085-2092, 2014. DOI: 10.1109/TED.2014.2318277

[14] M. Green, J. Zhao, A. Wang, P. Reece, and M. Gal, Efficient silicon light-emitting diodes, Nature, vol. 412, pp. 805-808, 2001. DOI: $10.1038 / 35090539$

[15] G. Piccolo, A. Kovalgin, R. Hueting, and J. Schmitz, Silicon LEDs in FinfET technology, Proc. ESSDERC, pp. 274-277, 2014. DOI: 10.1109/ESSDERC.2014.6948813

[16] F. Stellari, A. Ruggeri, H. Ainspan, and P. Song, Spontaneous photon emission from $32 \mathrm{~nm}$ and $14 \mathrm{~nm}$ SOI FETs, Proc. IEEE Int. Reliab. Phys. Symp., pp. 7B11-7B15, 2016. DOI: 10.1109/IRPS.2016.7574577

[17] E. F. Schubert, Light-Emitting Diodes, 2nd ed. Cambridge University Press; Cambridge, 2006.

[18] J. Schmitz, R. de Vries, C. Salm, T. Hoang, R. J. E. Hueting, and J. Holleman, On the switching speed of SOI LEDs, Proc. EUROSOI, pp. 101-102, 2008.

[19] V. Puliyankot, G. Piccolo, R. J. E. Hueting, A. Heringa, A. Y. Kovalgin and J. Schmitz, Increased light emission by geometrical changes in Si LEDs, Proc. Group IV Photonics, pp. 287-289, 2011. DOI: 10.1109/GROUP4.2011.6053792

[20] W. van Roosbroeck and W. Schockley, Photon-radiative recombination of electrons and holes in germanium, Phys. Rev., vol. 94(6), pp. 15581560, Jun. 1954. DOI: 10.1103/PhysRev.94.1558

[21] W. P. Dumke, Spontaneous radiative recombination in semiconductors, Phys. Rev., vol. 105(1), p. 139, Jan. 1957. DOI: 10.1103/PhysRev.105.139

[22] R. Hall, Ed., Recombination process in semiconductors, ser. Proc. IEE, no. 923-931, March 1960.

[23] Y. P. Varshni, Band-to-band radiative recombination in groups $I V, V I$, and III-V semiconductors (I), Phys. Stat. Sol. (b), vol. 19(2), pp. 459514, 1967. DOI: 10.1002/pssb.19670190202

[24] V. Puliyankot and R. J. E. Hueting, $1 D$ physical model to predict the internal quantum efficiency of silicon based LEDs, IEEE Trans. Electron Devices, vol. 59(1), pp. 26-34, Jan. 2012. DOI: 10.1109/TED.2011.2170992

[25] O. Heikkilä, J. Oksanen, and J. Tulkki, Ultimate limit and temperature dependency of light-emitting diode efficiency, J. Appl. Phys., vol. 105, no. 9, p. 093 119, May 2009. DOI: 10.1063/1.3125514

[26] T. Hoang, P. LeMinh, J. Hollemand and J. Schmitz, Strong efficiency improvement of SOI-LEDs through carrier confinement, IEEE Electron Device Lett. 28(5) pp. 383-385, 2007. DOI 10.1109/LED.2007.895415

[27] Le Minh Phuong, Silicon light emitting devices for integrated applications, Ph.D. Thesis, University of Twente 2003.

[28] XenICs infrared camera manual for users, 2006.

[29] Synopsys Sentaurus Device User Guide, Version a-2008.09 ed., 2009.

[30] D. B. M. Klaassen, A unified mobility model for device simulation, I. Model equations and concentration dependence, Solid State Electron. vol. 35(7), pp. 953-959, Jul. 1992. DOI: 10.1016/0038-1101(92)90325-7

[31] D. B. M. Klaassen, J. W. Slotboom, and H. C. de Graaff, Unified apparent bandgap narrowing in $n$ - and p-type silicon, Solid State Electron., vol. 35(2), pp. 125-129, Feb. 1992. DOI: 10.1016/00381101(92)90051-D

[32] M. Levinshtein, S. Rumyantsev, M. Shur, and Y. A. Porovskii, Eds Handbook Series on Semiconductor Parameters, Volume 1: $\mathrm{Si}, \mathrm{Ge}$, C (Diamond), GaAs, GaP, GaSb, InAs, InP, InSb. World Scientific, Singapore 1996.

[33] D. B. M. Klaassen, A unified mobility model for device simulationII: Temperature dependence of carrier mobility and life time, Solid State Electron., vol. 35(7), pp. 961-967, Jul. 1992. DOI: 10.1016/00381101(92)90326-8

[34] J. Lutz, Semiconductor power devices: physics, characteristics and reliability. Springer; Berlin, Heidelberg, 2011.

[35] S. M. Sze and M. -K. Lee, Semiconductor Devices, Physics and Technology 3rd ed., John Wiley and Sons Inc., New Jersey, 2013. 


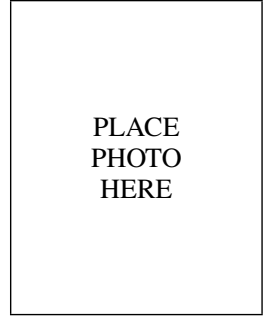

Vidhu Puliyankot received the B.Tech. degree from the Government College of Engineering, Kannur, India, in 2006 and the M.Sc. degree in nanoelectronics and nanomechanics from the University of Leeds, Leeds, U.K., in 2007. Between 2008 and 2012 he was a PhD student at the Semiconductor Components group of the MESA+ Institute for Nanotechnology, University of Twente, Enschede, The Netherlands.

His research interests comprise the design and optimization of light-emitting devices for Si technology platform. His research activities have included the modeling and optimization of second harmonic generation in asymmetric quantum-well devices.

\begin{tabular}{|c|} 
\\
\end{tabular}

Jurriaan Schmitz (M'02-SM'06) was born in Elst, The Netherlands in 1967. He received his M.Sc. (with honors) and Ph.D. in experimental physics from the University of Amsterdam (Amsterdam, The Netherlands) in 1990 and 1994 respectively, studying radiation imaging detectors for the Large Hadron Collider.

He was a CERN Summer Student in 1990. He joined Philips Research (Eindhoven, The Netherlands) in 1994 as Senior Scientist to work on CMOS device technology, characterization and reliability. In 2002 he was appointed as Professor of Semiconductor Components at the University of Twente (Enschede, The Netherlands) where he now leads the group of Integrated Devices and Systems. He (co)authored over 220 scientific papers and holds 18 patents. His research interests include semiconductor devices, microfabrication, thin film technology and wafer-level electrical characterization.

Prof. Schmitz is a member of the Dutch Physical Society NNV and the Dutch Vacuum Society NEVAC. For IEEE he served on the VLSI Technology and Circuits committee and the Ernst Weber Award committee. In 2008-2013 he chaired the Benelux Chapter of the IEEE Electron Device Society. He acted as Editor for IEEE Electron Device Letters in 2013-2017 and served in the technical program committees of several conferences including IEEE ICMTS, IRPS, IEDM and ESSDERC-ESSCIRC. He is a member of the Scientific Advisory Board of Solid State Electronics.

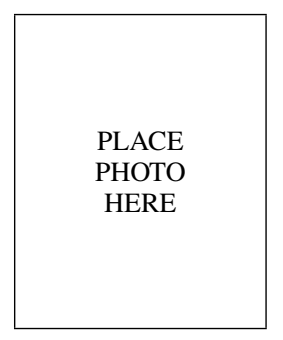

Giulia Piccolo was born in Pitigliano (GR), Italy. She received her BSc and MSc degrees in electronics from the University of Pisa, Italy in 2004 and 2006 respectively. After a research assignment at the Delft University of Technology she joined the University of Twente between 2007 and 2012 as a PhD student. Since 2013 she is presently a Technical Instructor and Training Developer at ASML, Veldhoven, The Netherlands.

\begin{tabular}{|c|}
\hline \\
PLACE \\
PHOTO \\
HERE \\
\end{tabular}

Raymond J.E. Hueting (S'94-M'98-SM'06) received the M.Sc. (with honors) and Ph.D. degrees in electrical engineering from Delft University of Technology, Delft, The Netherlands. His Ph.D. thesis dealt with the device physics of SiGe-based heterojunction bipolar transistors. In 1997 he joined Philips semiconductors, Nijmegen, the Netherlands where he worked on lateral power MOSFETs in SOI-based BCD-IC processes used for automotive applications.

He joined Philips research laboratories both Eindhoven, the Netherlands, and Leuven, Belgium, in 1998 respectively 2001 where he worked on device physics of trench-gate power MOSFETs, used for power supplies and automotive applications. He was also involved in the development of novel SiGe-based heterojunction devices. In 2005 he joined the Integrated Devices and Systems (former Semiconductor Components) group at the MESA+ Institute for Nanotechnology, University of Twente, the Netherlands, where he has been involved in the field of semiconductor device physics and modelling.

Dr. Raymond J.E. Hueting authored and co-authored over 80 papers and 35 US patents. He participates and participated in the technical programme committee of the ESSDERC and the ISPSD conference, respectively. 